\title{
COVID and Christ: Remote, Faith-Based Activities Using Religious Technology in the Catholic Church
}

\author{
Stephen J. McNeill \\ Kennesaw State University, USA
}

\begin{abstract}
Only 39 percent of American Catholics say they attend church in any given week (Gallup, 2019). In an effort to connect and engage the global congregation of the Catholic Church, the Vatican partnered with an external developer to create mobile learning tools-a project cumulating with the iOS and Android app "Click to Pray" and hardware extension app "eRosary." Click to Pray is the prayer platform of the "Pope's Worldwide Prayer Network" (PWPN), a pontifical work whose mission is to mobilize Catholics through prayer and action. Founded in 1844 as the Apostleship of Prayer, the PWPN is present in 98 countries and is made up of more than 35 million Catholics. This study aims to review the technology and security of the software; design of the hardware; the end-user experience, and the primary demographics of those who have downloaded either the Click to Pray or eRosary apps. This paper will also examine the idea of authenticity and replication of the religious experience in a remote atmosphere, in addition to its uses and gratifications. The eRosary is also capable of monitoring the health of the user, providing an interesting example of mobile learning device convergence for mind, body, and spirit.
\end{abstract}

Keywords: biometric user interfaces, wearable technology, religious technology, mobile learning, data privacy

\section{Introduction}

"You are dust and you will be dust..."

It was Ash Wednesday, February 26, 2020. The emergence of COVID-19 would soon change the nation and world forever. There was already talk within the church about social distancing; nevertheless, services still ran much as they always had, including the bestowing of ashes-and, even more alarmingly, handshake greetings between parishioners. By the time Easter Sunday arrived, there were no services to attend.

There have been at least three other major pandemics in the past century; however, none of them involved church closures-particularly, a closure during one of the most holy days of the Catholic calendar. Amidst a global pandemic, many Catholics have found themselves displaced, with most churches closed for services. Additionally, some parishioners are disabled, impaired, or without transportation. Now, certain religious acts are being examined anew in a digital atmosphere. 


\section{Literature Review}

The Vatican's 2020 Easter services were conducted remotely. The Pope and a small fraction of clerics, alone in the Basilica, prayed as cameras beamed and streamed the service to parishioners. While the Archdiocese of Philadelphia encouraged private praying to the rosary as a "personal time of prayer," the Pope's Worldwide Prayer Network's recently launched "Click to Pray" and the "Click to Play eRosary" as an alternative to solitary prayer for the more communally-minded parishioner. Advances in technology allow for more connectivity within a church community during a time when COVID-19 has eliminated the ability to worship in-person at the local Catholic church.

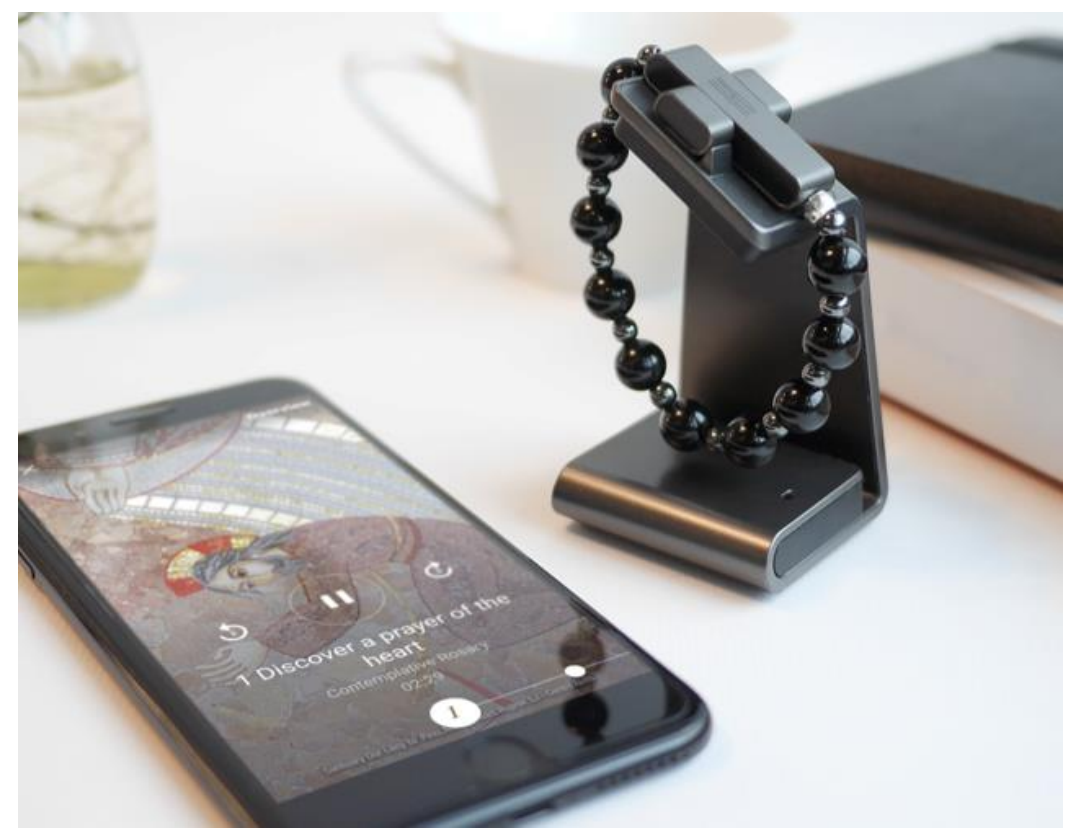

Figure 1. "Click to Pray eRosary" beads shown on charger with the companion app shown on the cell phone. Source: Pope's Worldwide Prayer Network, 2019.

\section{Click to Pray: background}

Click to Pray is the prayer platform of the Pope's Worldwide Prayer Network (PWPN), whose pontifical mission is to mobilize Catholics through prayer and action to face the challenges of humanity and the missions of the Church. These challenges are presented in the form of prayer intentions entrusted by the Pope to the Catholic community. Their mission is part of the dynamic of "The Heart of Jesus," a mission of compassion for the world, founded in 1844 as "The Apostleship of Prayer." The PWPN is present in 98 countries and is made up of more than 35 million Catholics, including the Eucharistic Youth Movement. The Pope established this ecclesial service in March 2018 as a pontifical work and approved its new statutes. Click to Pray invites everyone to pray for and act upon the challenges of humanity and the mission of the Church as identified by the Pope in his monthly intentions.

La Machi Group serves as communications agency hired by the PWPN to produce and manage their specific hardware and mobile application projects. La Machi Group led the design, digital 
development, digital marketing, community management and press and promotion campaign. Moreover, La Machi Group partnered with GTI (Acer, Inc.) for the hardware execution of eRosary, culminating in a product that also has an integrated fitness tracker with six-axis inertial sensing. The eRosary bracelet is also IP67 water and dust resistant and connects using Bluetooth 5.0. The hardware itself contains a $15 \mathrm{mAh}$ lithium-ion battery and offers wireless charging on a proprietary cradle.

\section{Mission and intention of the Click to Pray application}

The purpose of the Click to Pray app is to connect people around the world through prayer. Each user with an account has the opportunity to share intentions with the 'Prayer Wall' feature, allowing users to submit intentions which are then shared with all members of the community for prayer. When the user clicks Click to Pray, one prayer is added to that intention. Generally, users share personal intentions, but there are also registered prayers for human tragedies or events that may have recently occurred. In fact, the 'Daily Prayer' is the app's most popular feature; for example, there were many reported prayer intentions for the humanitarian crisis in Venezuela. What seems most remarkable is that this specific intention not only appeared in Spanish, but is translated into other common languages, too, highlighting Click to Prey's main purpose and slogan: connect your prayer with the world.

\section{Demographic information of active locations worldwide}

\section{Website}

1.a. Top three user countries (since the launch):

United States: $19.07 \%$

Brazil: $10.68 \%$

Italy, 10.12\%

1.b. Top three user languages (since the launch)

English: $28.13 \%$

Spanish: $27.38 \%$

Portuguese: $23.4 \%$

\section{App}

2.a Top three countries

Italy: $22.1 \%$

Portugal: $16 \%$

United States: $14.3 \%$

\section{Facebook}

3.a User language:

English: 98,596

Spanish: 25,744

French: 31,500

Portuguese: 38,659 
German: 539

Italian: 3,767

Chinese: 529

\section{Twitter}

4.a User language:

Spanish: $51 \%$

English: 45\%

Portuguese: $17 \%$

French: $7 \%$

Italian: 6\%

\section{Instagram}

\section{5.a Top five user countries}

Brazil: 15\%

Portugal: $11 \%$

USA: $6 \%$

Colombia: $5 \%$

Argentina: 5\%

\section{Technology}

La Machi Group identified the target audiences of "Click to Pray," dividing them into three types:

1. according to the message

2. according to the countries

3. according to their relationship with the application

Based on the aforementioned types, researchers then created different profiles. To better understand the User Experience (UX), La Machi Group examined users' age, sex, knowledge of the project, and whether old or new users. The results of that study helped La Machi Group identify points of improvement in the UX.

The mobile application Click to Pray is a non-proprietary native build. Upon the launch, the initial downloads totaled over 520,000:

$$
\begin{array}{ll}
\text { - } & \text { Android 318,534 } \\
\text { - } & \text { iOS 202,324 } \\
\text { - } & \text { Total: 520,858 }
\end{array}
$$

Within the first six months, Click to Pray reached 2,000,000 members among its social networks, app users, newsletter subscribers, and website visits. Of smartphone users, $64 \%$ were Android and $36 \%$ iOS. The number of subscribers continues to increase daily.

\section{Other Catholic mobile applications}

The Catholic Church and Vatican City previously used mobile applications available within the City State and Holy See. They are officially published by the Segreteria per la Communicazione 
(Communication Secretary). Among them are companion apps for Vatican.va, L'Osservatore Romano (the newspaper for the Holy See), Radio Vaticana, and weekly Homilies.

\section{Methodology}

\section{Location of study}

The current study was conducted on or near campus at Kennesaw State University, as well as online through computer-aided research. The location was chosen because the researchers conducting the study live and work near Kennesaw State University, making participation more accessible.

\section{Research design and sampling procedure}

The study took place for 30 continuous days from April 1-30, 2020. The timeframe was intentionally chosen because it was long enough to chart progress and to see positive outcomes within the userinterface-as well as to examine potential problematic situations within the app and hardware, if applicable.

\section{Data collection}

Data was gathered for specific themes and trends, including commonalities between Click to Pray and its associated eRosary mobile applications. The data gathered was both qualitative and quantitative. Certain measurements were made regarding specific Uses and Gratifications.

\section{Data-gathering instruments}

1. Privacy: within Terms of Service.

2. Efficacy and usability: ease of use.

3. Comparison to traditional worship: Application or replication of authentic experience(s) during use.

4. User Education: measured data presented to users during use.

\section{Data Analysis}

Focusing on each measurement, the current study analyzed and organized the gathered data into specific categories to best examine the effects of the Click to Pray mobile applications; it also examined negative and positive data in relation to the total testing period while recognizing and charting relevant patterns and themes in the study's results.

\section{Results}

Focusing each measurement, the researchers analyzed the gathered data and organized it into specific categories to best examine the usability of the Click to Pray and eRosary mobile learning applications, including examining negative and positive data in relation to the total testing period (April 1-30, 2020):

\section{Privacy: including terms of service.}

\section{Positive outcomes:}

Users are afforded the option of praying directly with the Pope, or with fellow users through the social network. Moreover, the app enables users to discover prayer intentions through 
the 'Prayer with the Network' and 'Pray Every Day' features (morning, afternoon, night). Users can also connect with their friends through the intentions and requests that they make. There are prayer campaigns and donation information as well.

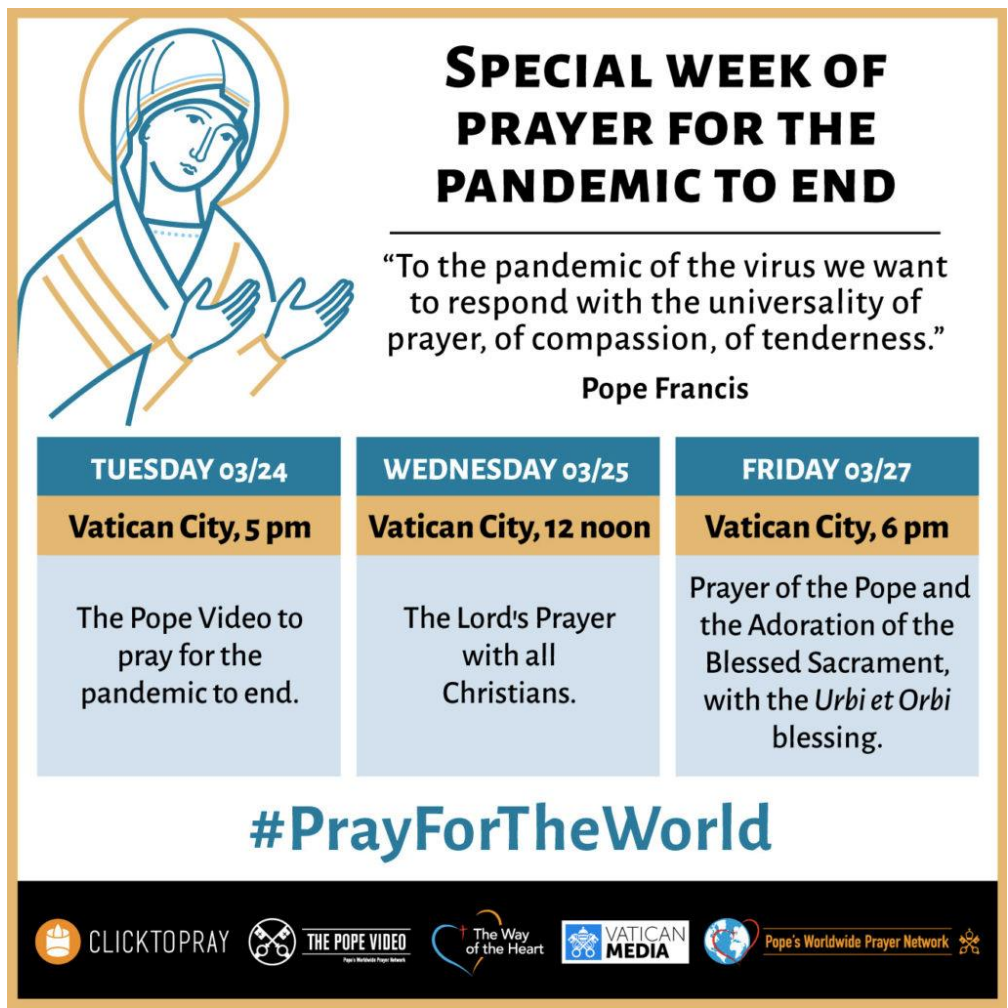

Figure 2. This figure depicts the online notice when Pope Francis issued "Special Week of Prayer" to end the pandemic. Source: Click to Pray app, 2020.

Potential negative outcomes:

- A user cannot interact with the Click to Pray app unless they sign up with their Facebook, Google, or email accounts and they must also enter into the Terms of Service.

\section{Efficacy and Usability}

\section{Positive outcomes:}

- Click to Pray has a list of tasks that the user can select before undertaking activities such as praying with the network, praying with the pope, and specific prayer campaigns. 

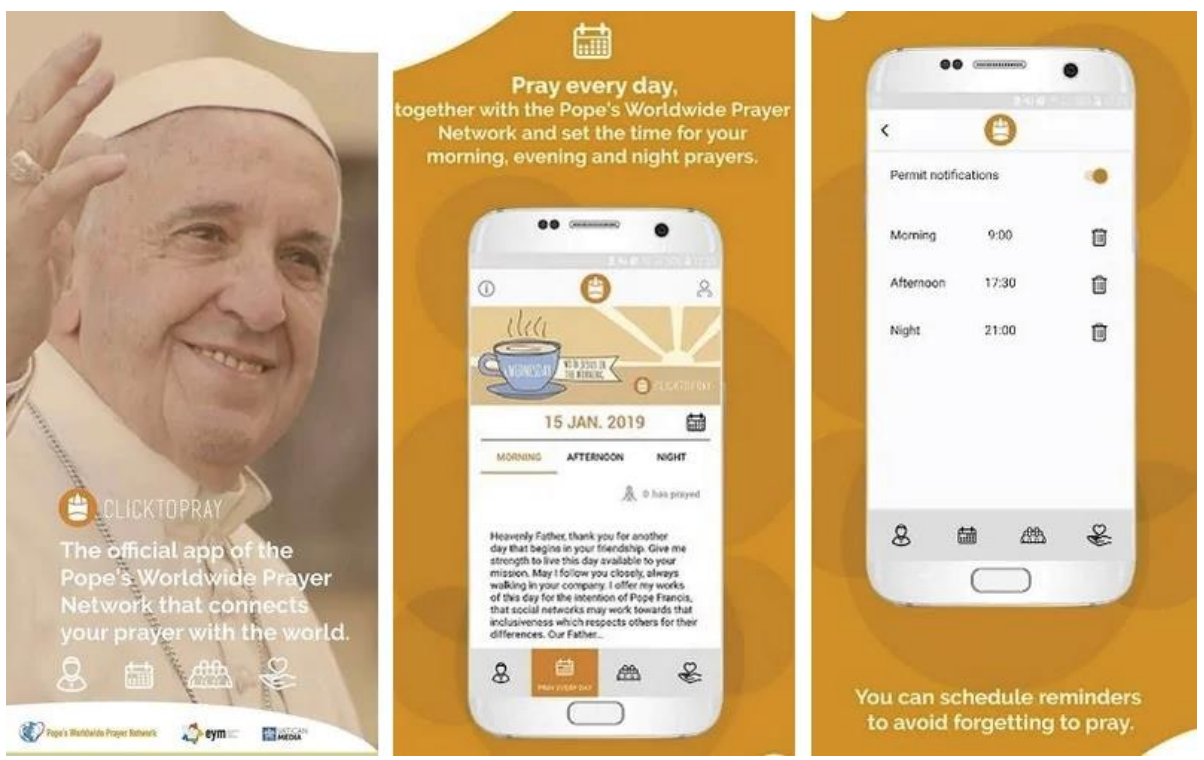

Figure 3. Scheduling reminders to pray shown within the app. Source: http://jesuits.ca/newsdetail?TN=NEWS-20190123125348, 2019.

- Click to Pray also offers a scheduled reminder to pray, with pop-up notifications when it is time to pray to the rosary.

- Users can customize health goals, such as steps per day. The eRosary will vibrate when the health goals are achieved, as well as incoming calls on a connected device.

- The eRosary bracelet also includes statistical information for rosaries prayed, complete rosaries prayed, and the total amount of time praying.

Potential negative outcomes:

- The eRosary hardware must be charged on a separate charging cradle daily (it also has USB connectivity).

- The eRosary issues a prayer notification twice a day, every day. By default, the application reminds the user in the morning and evening, which may prove potentially inconvenient for those who have not customized their reminders on the app. Notifications can be turned off under settings.

- The Bluetooth connection can sometimes exhibit latency issues if the phone is housed in a case. However, the Bluetooth does not have to be connected at all times as the eRosary will update the data with the companion app once it is reconnected. 


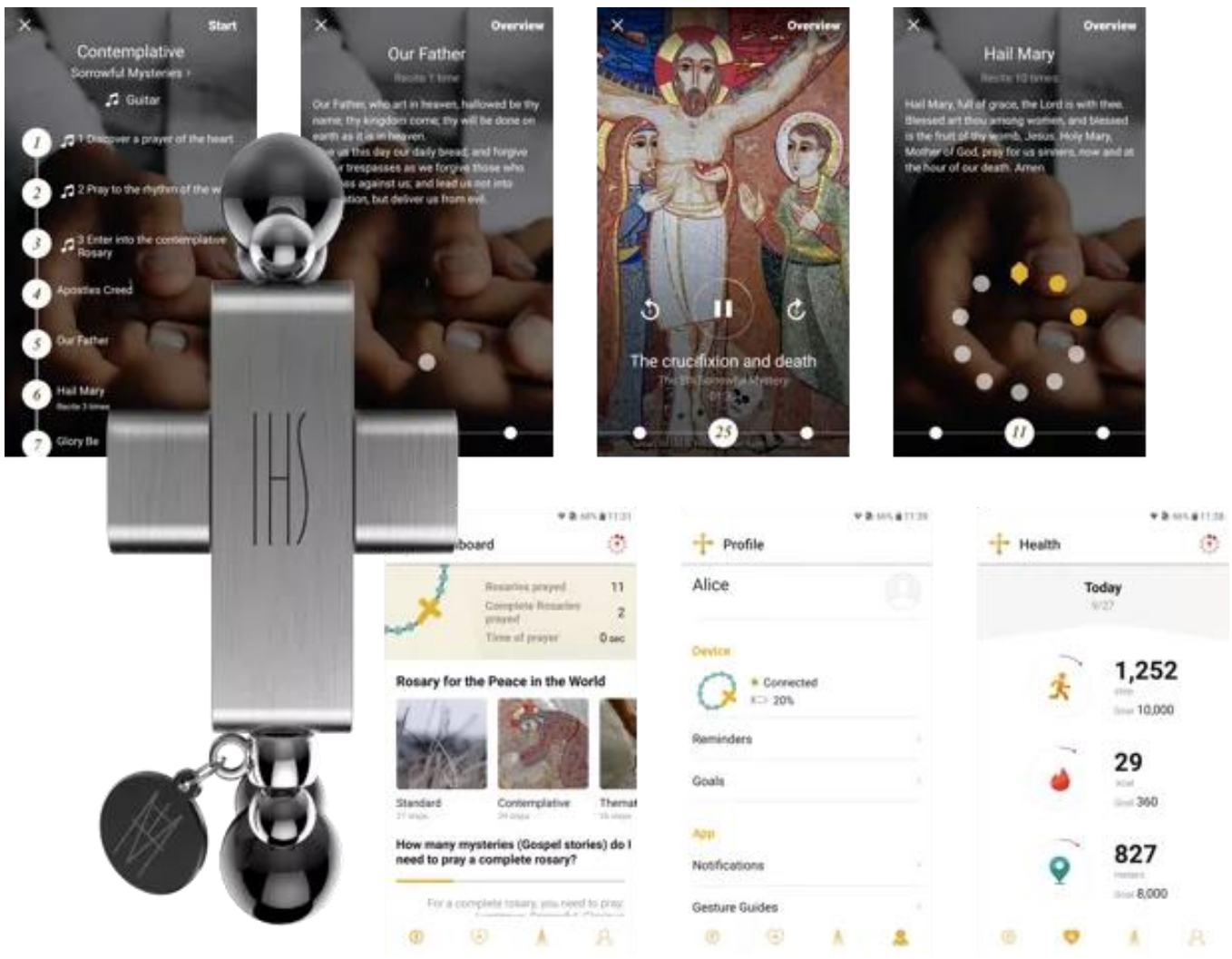

Figure 4. Screenshots displaying eRosary's mobile learning features. Source:

https://erosary.clicktoprayerosary.org/en-us/index.html, 2019.

\section{Comparison to traditional worship: Application or replication of authentic experience(s) during use.}

\section{Positive outcomes:}

- The eRosary was designed to duplicate the authentic experience of traditional rosary prayer for the user. The physical movements of rosary prayer are duplicated, helping to educate the new user on how to pray to the rosary with the corresponding mobile application.

- The eRosary hardware is constructed to be worn as a bracelet and manufactured with the look and feel of a traditional rosary. The device is outfitted with black agate and hematite rosary beads, and a 'smart cross,' which stores the data connected to the associated application.

\section{Potential negative outcomes:}

- To unlock eRosary, the user is required to hold the beads and tap the four positions of your body to make the sign of the cross. There was difficulty in duplicating success in unlocking the hardware multiple times without issue.

- The user must shake the eRosary twice to move to the next prayer steps, which was also problematic. 
- The corresponding app does not encourage engagement when praying to the rosary-it serves only as a 'reminder' message.

\section{User education: measured data presented to users during use.}

\section{Positive outcomes:}

- The eRosary mobile application successfully gamifies the rosary, with daily encouragements for success with messages such as "For a complete rosary, you need to pray: Joyful, Luminous, Glorious," which appear on the application's corresponding dashboard.

- The corresponding eRosary app can integrate with eRosary devices, doubling as a fitness tracker providing detailed information for the user when worn daily. This data includes steps, calories burned, and meters walked per day, week, month, and year. Moreover, users can access all recorded data, which is displayed in half-hour increments.

\section{Potential negative outcomes:}

- The eRosary must be worn in order to register the prayers on the corresponding app ("Click to Pray's eRosary"). Many times, the connection drifted, not allowing the Rosary to update the fitness tracker feature.

- The eRosary measures biometric data from three areas: steps, calories burned, and meters walked per day. The mobile application does not contextualize the data nor show any graphical representation of progress beyond the daily total. The user can toggle between dates, however.

\section{Discussion}

Only 39 percent of American Catholics say they attend church in any given week. With this data in mind, the Pope's Worldwide Prayer Network collaborated with La Machi Group, who, in partnership with GTI (Acer Group), created the Click To Pray app and the Click To Pray eRosary in order to facilitate engagement, connectivity, and mobile learning. This paper discusses application topics including the background of Click to Pray and the eRosary mobile applications, also reviewing similar Vatican mobile applications as well as user education, uses and gratifications.

\section{Comparisons to Previous Research}

While there have been no 'officially released' academic or consumer trials of this product, consumer statistics from La Machi Group are available online. The applications have mixed product reviews from consumers, showing no outstanding public opinions either way. Several reviewers, however, noted the sophistication of the eRosary from a hardware perspective; other reviews noted that the eRosary's privacy may have been compromised due to hacking within hours of the initial release (October 2019).

\section{Limitations of the Study}

There were no focus groups or other large-scale tests of the product. The study was limited to discovery research based on the criteria outlined in the methodology and results sections of this paper. Moreover, there was no way to measure multiple-user, self-reported success with learning to pray to the rosary. 


\section{Suggestions for Future Research}

There should be a large-scale study with pre- and post-testing of knowledge about users' backgrounds and abilities to chart progress in learning how to pray to the Rosary by using the eRosary as a mobile learning tool. Moreover, an analysis of vulnerability of the data and associated privacy concerns should be examined in the context of the hardware and software.

\section{Conclusion}

The Click to Pray and eRosary mobile applications allow users to monitor their health and remain committed to praying to the rosary while engaged in various tasks inside and outside the home. Catholics affected by the COVID-19 pandemic have an option to ease the struggles of social isolation and self-isolation measures. The Click to Pray and eRosary mobile applications provide the benefit of using mobile learning technology for teaching how to pray to the rosary and reminders for prayer that can be controlled by the user. The hardware and software do have several drawbacks but they do not outweigh the benefits dependent on situational analysis and user needs.

\section{References}

[1] Chu, H. (2019, October 16). The Vatican wants you to pray with this smart rosary. Retrieved July 12, 2020, from https://mashable.com/article/smart-rosary/?europe=true

[2] Click to Pray eRosary - Press Kit Shared Folder. (n.d.). Retrieved July 12, 2020, from https://drive.google.com/open?id=1-YOVq5N5XNFvcM3AXITNpUqST2ikVe0k

[3] "Click to Pray eRosary" - wearable smart device to pray the rosary for peace. (2019, October 15). Retrieved July 12, 2020, from https://www.vaticannews.va/en/vaticancity/news/2019-10/click-pray-rosary-smart-digital-device-world-peace.html

[4] Hollister, S. (2019, October 18). Does praying burn calories? Here's the pope's \$110 erosary. Retrieved July 12, 2020, from https://www.theverge.com/circuitbreaker/2019/10/17/20920101/click-to-pray-erosary-catholic-church-vatican-prayer-beads

[5] Meisenzahl, M. (2020, March 18). As the coronavirus spreads, Catholics are turning to online spiritual practices, from masses live-streamed from the Vatican to a \$110 wearable 'eRosary'. Retrieved July 12, 2020, from https://www.businessinsider.com/vatican-wearable-erosary-young-catholics-prayer2019-10? IR=T

[6] Pope makes special request for prayers for the sick. (2020, March 26). Retrieved July 12, 2020, from https://www.vaticannews.va/en/pope/news/2020-03/pope-makes-specialrequest-for-prayers-for-the-sick.html

[7] Rogers, J. (2019, October 17). Vatican unveils 'smart rosary' activated by making the sign of the cross. Retrieved July 12, 2020, from https://www.foxnews.com/tech/vaticanunveils-smart-rosary

[8] Saad, L. (2020, April 08). Catholics' Church Attendance Resumes Downward Slide. Retrieved July 12, 2020, from https://news.gallup.com/poll/232226/church-attendanceamong-catholics-resumes-downward-slide.aspx

[9] Sorto, G. (2019, October 16). Vatican launches $\$ 110$ 'click to pray' wearable rosary. Retrieved July 12, 2020, from https://edition.cnn.com/2019/10/16/world/vatican-clickto-pray-erosary-trnd/index.html 
[10] Spagnuolo, E. (2019, October 16). ERosary, ora puoi pregare con il rosario smart. Retrieved July 12, 2020, from https://www.wired.it/gadget/accessori/2019/10/16/erosary-rosario-smart-tech/

[11] Staff, C. (2020, March 20). Coronavirus in Philadelphia: Archbishop Nelson Perez Says Now Is 'Time to Go Into Our Personal Prayer Life'. Retrieved July 12, 2020, from https://philadelphia.cbslocal.com/2020/03/20/coronavirus-in-philadelphia-archbishopnelson-perez-says-now-is-time-to-go-into-our-personal-prayer-life/

[12] Staff, F. (2019, October 18). Vaticano lanza rosario electrónico que se activa haciendo la señal de la cruz • Forbes México. Retrieved July 12, 2020, from https://www.forbes.com.mx/vaticano-lanza-rosario-electronico-que-se-activa-haciendola-senal-de-la-cruz/

[13] Wilson, M. (2019, October 17). The pope's first wearable is a $\$ 110$ rosary that tracks your prayers. Retrieved July 12, 2020, from https://www.fastcompany.com/90417708/the-popes-first-wearable-is-a-110-rosarythat-tracks-your-prayers 